University of Wollongong

Research Online

Faculty of Engineering - Papers (Archive)

Faculty of Engineering and Information

Sciences

$1-1-2012$

\title{
A simple and cost-effective method for fabrication of integrated electronic- microfluidic devices using a laser-patterned PDMS layer
}

\author{
Ming Li \\ University of Wollongong, ml433@uowmail.edu.au \\ Shunbo Li \\ The Hong Kong University of Science and Technology \\ Jinbo Wu \\ The Hong Kong University of Science and Technology \\ Weijia Wen \\ The Hong Kong University Of Science And Technology \\ Weihua Li \\ University of Wollongong, weihuali@uow.edu.au
}

See next page for additional authors

Follow this and additional works at: https://ro.uow.edu.au/engpapers

Part of the Engineering Commons

https://ro.uow.edu.au/engpapers/4519

\section{Recommended Citation}

Li, Ming; Li, Shunbo; Wu, Jinbo; Wen, Weijia; Li, Weihua; and Alici, Gursel: A simple and cost-effective method for fabrication of integrated electronic-microfluidic devices using a laser-patterned PDMS layer 2012, 751-760.

https://ro.uow.edu.au/engpapers/4519

Research Online is the open access institutional repository for the University of Wollongong. For further information contact the UOW Library: research-pubs@uow.edu.au 


\section{Authors}

Ming Li, Shunbo Li, Jinbo Wu, Weijia Wen, Weihua Li, and Gursel Alici 


\title{
A simple and cost-effective method for fabrication of integrated electronic- microfluidic devices using a laser-patterned PDMS layer
}

\author{
Ming $\mathrm{Li}^{1}$, Shunbo $\mathrm{Li}^{2}$, Jinbo Wu${ }^{3}$, Weijia Wen*², ${ }^{2,3}$, Weihua $\mathrm{Li}^{*}{ }^{1}$, Gursel Alici \\ ${ }^{1}$ School of Mechanical, Materials and Mechatronic Engineering, University of Wollongong, \\ Wollongong, 2522 NSW, Australia \\ ${ }^{2}$ Department of Physics, The Hong Kong University of Science and Technology, Clear Water \\ Bay, Kowloon, Hong Kong \\ ${ }^{3}$ Nano Science and Technology Program and KAUST-HKUST Micro/Nanofluidic Joint \\ Laboratory, The Hong Kong University of Science and Technology, Clear Water Bay, \\ Kowloon, Hong Kong \\ *E-mails: phwen@ust.hk; weihuali@uow.edu.au \\ Phone: + 8522358 7979; +612 42213490 \\ Fax: +852 2358 1652; +61242213238
}

\begin{abstract}
We report a simple and cost-effective method for fabricating integrated electronic-microfluidic devices with multilayer configurations. $\mathrm{A} \mathrm{CO}_{2}$ laser plotter was employed to directly write patterns on a transferred polydimethylsiloxane (PDMS) layer, which served as both a bonding and a working layer. The integration of electronics in microfluidic devices was achieved by an alignment bonding of top and bottom electrode-patterned substrates fabricated with conventional lithography, sputtering and lift-off techniques. Processes of the developed fabrication method were illustrated. Major issues associated with this method as PDMS surface treatment and characterization, thickness-control of the transferred PDMS layer, and laser parameters optimization were discussed, along with the examination and testing of bonding with two representative materials (glass and silicon). The capability of this method was further demonstrated by fabricating a microfluidic chip with sputter-coated electrodes on the top and bottom substrates. The device functioning as a microparticle focusing and trapping chip was experimentally verified. It is confirmed that the proposed method has many advantages, including simple and fast fabrication process, low cost, easy integration of electronics, strong bonding strength, chemical and biological compatibility, etc.
\end{abstract}

Key words:

Microfluidics, Electronics integration, PDMS, Laser ablation, Transfer bonding, Dielectrophoresis

\section{Introduction}

The common fabrication of microfluidic chips involves two major issues: construction of open channel and bonding of machined substrates to form an enclosed structure. Open channels can be obtained by etching in silicon or glass using standard MEMS technique (Harrison et al. 1992; Jacobson et al. 1994). However, the etching step is expensive, relatively slow and/or using aggressive chemicals. Moreover, glass and silicon bonding techniques generally require stringent conditions, which are not always compatible with electronic wafers. For 
example, high voltage (200-1,500V) and high temperature $\left(150-500^{\circ} \mathrm{C}\right)$ are required by anodic bonding (Tong and Gösele 1999; Iyer and Auberton-Hervé 2002; Alexe and Gösele 2004); the process of fusion bonding (Tong and Gösele 1999; Iyer and Auberton-Hervé 2002; Alexe and Gösele 2004) needs ultra clean surfaces and high bond temperature $\left(600-1,200^{\circ} \mathrm{C}\right)$; solder bonding (Sparks et al. 2001) and thermocompression bonding (Maharbiz et al. 1999) involve deposition and patterning of additional layer, and heat or pressure for close contact of substrates. Although femtosecond laser has been employed to create enclosed microfluidic structures inside transparent chips without bonding, this method is relatively expensive and not suitable for mass production (Cheng et al. 2004, 2008). Significant advances in the approaches for fabricating microfluidic chips have made with the introduction of soft lithography (Xia and Whitesides 1998; Quake and Scherer 2000; McDonald et al. 2000; Wu et al. 2003), laser ablation (Roberts et al. 1997; Schmidt et al. 1999; Snakenborg et al. 2004), X-ray lithograph (Ueno et al. 2000; Ford et al. 1999) for creating polymer microchannels, and adhesive bonding technique (Schmidt 1998; Kovacs 1998) for assembly. These have advantages including cost reduction (in terms of time, labor, and facilities), low bonding temperature (depending on the polymer material between room temperature and $450^{\circ} \mathrm{C}$ ), ability to join practically any wafer materials without special surface treatment as planarization and excessive cleaning. Among those polymers applied for fabricating microfluidic devices, PDMS is particularly appealing, because it is transparent, flexible, replicable, inexpensive, biocompatible, and conformal with various substrates (Xia and Whitesides 1998; Whitesides 2006; El-Ali et al. 2006)

However, there is still a challenge to combine multiple existing structured microfluidic parts together, especially when packaging microfluidic devices that integrating electronic elements on both top and bottom surfaces of microfluidic channels for more advanced functions. Wu et al. (2005) used a thin layer of PDMS prepolymer as an adhesive for the formation of microfluidic devices of various materials, but this method requires an exact control of the thickness of PDMS prepolymer layer, because the reflow of the PDMS prepolymer may deform the initial geometry of the channel or even block narrow channels if the adhesive layer is relatively thick. Zhang et al. (2010) reported using transferred PDMS layers to fabricate PDMS multilayer microfluidic chips, however, materials excluding PDMS were not discussed, repetitive mold-replica fabrication processes were required for every patterned PDMS layer, and this method is not suitable to integrate electronic components in microfluidic chips. Plecis and Chen (2007) proposed the use of plasma-treated PDMS layer for bonding two glasses with $\mathrm{SiO}_{2}$ and $\mathrm{Si}_{3} \mathrm{~N}_{4}$ coating on the surfaces, however, the microfluidic channel construction process required photolithography for sacrificial template, and additional wet etching or reactive ion etching (RIE) were needed to remove residual PDMS layer. Such fabrication processes are complicated, timeconsuming, typically associated with strict conditions, and not suitable for common laboratorial environment.

In order to overcome the drawbacks mentioned above, we defined and machined open channels on a transferred thin layer of PDMS using a direct-write, mask-free laser ablation technique, which is followed by transferring, aligning, and bonding the laser-patterned PDMS layer on patterned substrates to form enclosed microfluidic channels. Incorporating electronics on chips was also achieved by 
surface patterning on the top and bottom substrates, and then plasma-bonding to the transferred PDMS layer. This method is simple and cost-effective due to the elimination of the demands for template and corresponding fabrication process, facilities and consumables in clean room. By integrating electronic components, the fabricated microfluidic device is not be simply a network of microchannels, but a multifunctional microsystem which is able to conduct advanced functions such as chemical or biological analysis and testing related to electronic processing and detection. In addition, devices with multilayer configurations can be obtained by repetitively plasma-bonding many laser-patterned PDMS layers together with the substrate, even more complex microfluidic systems having channels of different depths can be formed by varying spin speed to control the thickness of transferred PDMS layers. Since PDMS is flexible and compatible, this method can be applied to fabricate devices of various substrates (glass, silicon, metal, polymer, etc) regardless of the flatness of the surface. In this paper, key fabrication processes were described. The validation of this method was conducted by examining and testing the bonding of glass-PDMS-glass and glassPDMS-silicon. As an application example, a dielectrophoresis (DEP)-based integrated electronic-microfluidic chip for focusing and trapping microparticles was fabricated and tested.

\section{Fabrication process}

Figure 1 shows the schematic flow of the fabrication process, which is made possible by a transfer process of a laser-patterned PDMS layer and oxygen-plasma bonding method for combining PDMS with two substrates. The overall fabrication approach for an integrated electronic-microfluidic device consists of four major steps: (1) PDMS layer processing, (2) microfluidic channel patterning, (3) substrate processing, and (4) bonding.

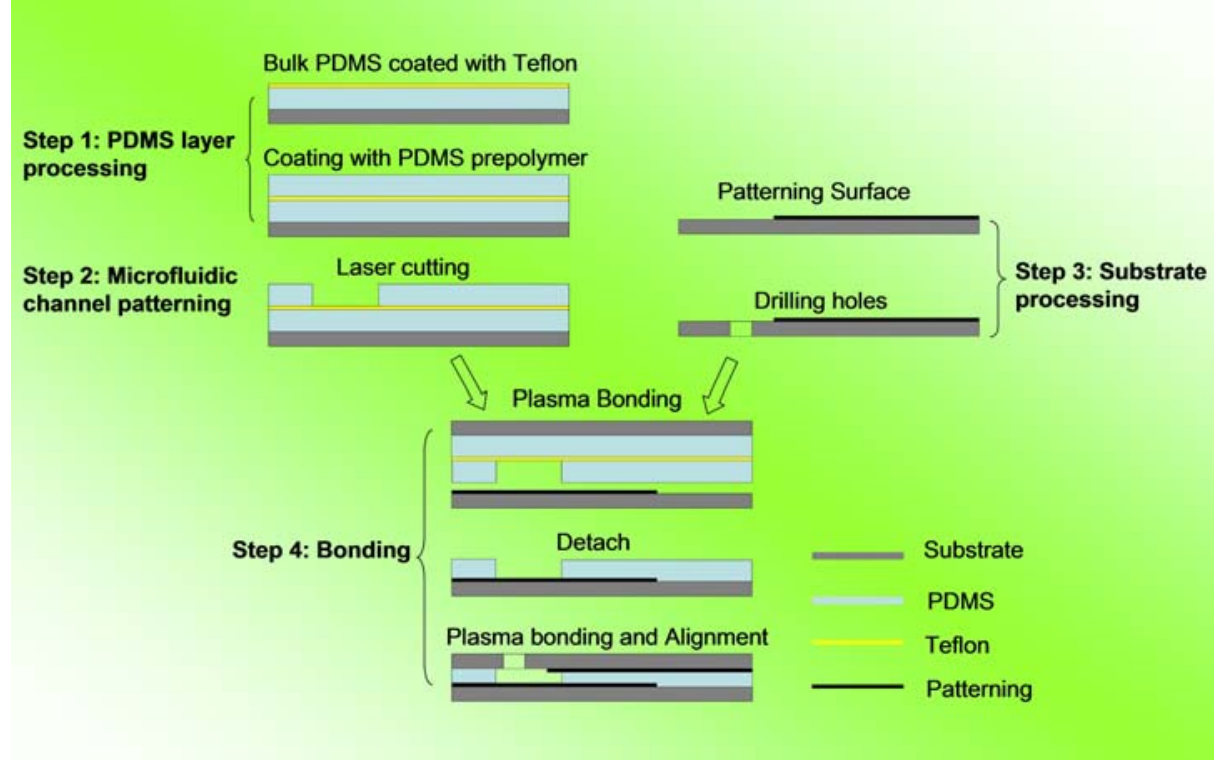

Fig. 1 Schematic view of fabrication process flow (diagram not to scale)

\section{PDMS layer processing}

The first step involves the preparation of two PDMS layers on a glass substrate: one bulk layer serves as a transferring layer after surface treatment, while the 
other thin layer is a working component of the device, which is also used as an adhesive for bonding with substrates. The transparent and flexible transferring PDMS layer ensures effective alignment and little deformation of the thin PDMS layer. A bulk PDMS slab is produced by Sylgard 184 silicone elastomer mixture (Dow Corning Corporation, Miland, USA) at a weight ratio of base: curing agent $=10: 1$. The mixture was volume-controlled poured onto a leveled glass surface, placed in a vacuum chamber to get rid of bubbles, and then put into an oven at $60^{\circ} \mathrm{C}$ for about $2 \mathrm{~h}$. After solidification, the upper surface of the bulk PDMS slab was treated by spin-coating a layer of Teflon AF solution (Dupont, Grade 601S1100-1), and then the slab was relaxed in room temperature for about $5 \mathrm{~min}$. A thin PDMS layer were generated by coating PDMS prepolymer on the prepared bulk PDMS with Teflon covering the surface, followed by heating on a hotplate at $60^{\circ} \mathrm{C}$ for curing. Thin PDMS layers of different thickness can be achieved by varying the spin speed of the spin coater. At the end of this step, a glass substrate with a two-layer PDMS slab having a Teflon interlayer was ready for laser cutting.

\section{Microfluidic channel patterning}

A $\mathrm{CO}_{2}$ laser plotter (Versa Laser System, Model VLS3.50, Universal Laser System, Ltd.) was employed to construct structures on the thin PDMS layer. It has the maximum power of 25W, maximum pulses per inch (PPI) of 1,000, and scanning speed ranging from 0.25 to $25 \mathrm{~mm} / \mathrm{s}$. A standard 2.0" focus lens is accompanied with the system, and the estimated focal spot size is around $50 \mu \mathrm{m}$. The designed pattern was drawn by AutoCAD software (Autodesk Inc., USA). After exporting the drawing to the drive which control the movement of laser, resetting scan mode and the value of parameters, and adjusting focus point, the required pattern was plotted directly on the thin PDMS layer. Such parameters as the laser power, scanning speed and pulses per inch (PPI) were optimized to cut through the thin PDMS layer and obtain patterns of ideal profile. Unwanted part of the thin PDMS layer was peeled off from the bulk PDMS slab having Tefloncoated surface using a tweezer, after which PDMS was cleaned using isopropanol to eliminate ashes and debris created by $\mathrm{CO}_{2}$ laser. In this step, open channel regions were defined on the transferred PDMS layer, which was bonded to suitable substrates to form enclosed microchannels later. This photoresist residuefree step eliminates clean room facilities, which were unlikely to be accomplished by other earlier-mentioned bonding methods.

\section{Substrate processing}

This step consists of patterning the top and bottom substrates. The materials of the substrate can be silicon, glass, plastic or others that can be well bonds to PDMS after surface pretreatment with plasma. Metal for electrodes, such as $\mathrm{Cu}, \mathrm{Au}$, and Pt, dielectrics or semiconductors including $\mathrm{SiO}_{2}, \mathrm{Al}_{2} \mathrm{O}_{3}, \mathrm{Si}_{3} \mathrm{~N}_{4}$ and $\mathrm{SiC}$, as well as organic materials for hydrophilic/hydrophobic modification and bioreagent can be grafted on the surface for different applications. Wet etching or reactive ion etching (RIE) technique can also be applied to construct defined patterns on the substrate. Holes serving as the inlet and outlet of the fluid were drilled in the top substrate by a drilling machine (DREMEL 220 workstation), and then the substrate was cleaned in an ultrasonic acetone bath, rinsed in DI water and isopropanol, and dried under nitrogen flow. By this step, electronic components 
can be integrated in the fabricated microfluidic devices, which is helpful for further chemical or biological testing and analysis, and to realize all-in-one microdevices.

\section{Bonding}

The bonding step was finally performed between the PDMS layer containing microfluidic channels and the top and bottom patterned substrates. One patterned substrate and the whole structure prepared after peeling off unwanted part from the laser-patterned PDMS layer were oxygen plasma treated in a plasma cleaner (Harrick Plasma PDC-002) for 2 min, after which the surface were covered with methanol acting as a lubricant for effective alignment, aligned under a microscope (Meiji Techno, 43660) and contacted face to face. After heating at $90^{\circ} \mathrm{C}$ and pressing with $50 \mathrm{~kg} / \mathrm{m}^{2}$ for $5 \mathrm{~min}$, the transferring PDMS slab was taken off, leaving the thin patterned PDMS layer well attached to the substrate. One key point of this bonding process is handling the thin laser-patterned PDMS layer with bulk PDMS slab and substrate, rather than a single thin film. Plasma bonding ensures a good adhesion between the plasma-treated substrate and the transferred PDMS layer, while the adhesion between two PDMS layers is reduced by the Teflon layer. Hence, the patterned PDMS layer is strongly stick to the substrate, and the dimension of the pattern could be preserved. Using the same plasmatreated process, the reverse side of the patterned PDMS layer was bonded with the other substrate. Thus, PDMS construction acting as an adhesive layer was converted to a microfluidic device integrating electronic component, and an enclosed microfluidic system was formed.

The fabrication approach discussed here employs two relatively recent advances in microfluidic device microfabrication: (1) a modified transfer process, in which a transferred PDMS layer is processed on the top of Teflon-treated bulk PDMS slab, as if it was an independent layer, which is then aligned, bonded, and released to chip; (2) laser ablation of polymer, in which microfluidic channels were directly and quickly constructed on PDMS. The fabrication technology taking advantage of transfer process and laser through-cutting method for integrating microelectrodes in microfluidic devices is described here for the first time. By repeating the microfluidic channel patterning and bonding steps, combining many patterned PDMS layers together with substrates can be achieved. This developed method provides a simple and fast technique to package integrated microfluidic devices having multiple layers and electronic components.

\section{Results and discussion}

\section{PDMS surface treatment}

A critical requirement of the transferring bulk PDMS slab is that the patterned PDMS layer can be easily taken off from it after bonding with the substrate. In order to detach two PDMS layers, the adhesion strength at their interface should be lower than the bonding force of plasma-treated PDMS and substrates. In order to reduce the bonding force between two PDMS layers, Teflon AF solution was spun on the surface of transferring bulk PDMS slab. The reasons of choosing Teflon for surface treatment are: (1) its transparency makes effective alignment 
possible, and (2) surface treatment using Teflon can be achieved by spin-coating it on the required surface, since it can quickly evaporate and its limited solubility (315\%) allows ultrathin coating in the submicron thickness range remaining on the surface. This process is simple, time-saving, and does not need additional complicated equipment as encapsulated chamber employed by Zhang et al. (2010) for PFOCTC deposition.

\section{Surface characterization}

The measurement of contact angle as well as X-ray photoelectron spectroscopy (XPS) analysis were performed to ensure that Teflon AF was coated on the surface of the transferring PDMS layer. The water contact angles were measured on the surfaces of bare PDMS and Teflon-treated PDMS by the sessile drop technique, and then analyzed using drop shape analysis software Image J. Three measurements were made and averaged. As can be seen in Fig. 2, the measured contact angles of bare PDMS (Fig. 2a) and Teflon-treated PDMS (Fig. 2b) were $101.1^{\circ}$ and $111.9^{\circ}$, respectively. The reason why the contact angle increased after surface treatment is due to the higher hydrophobicity and roughness of Teflon. The increase of contact angle after surface treatment confirming the successful coating of Teflon on the PDMS surface.

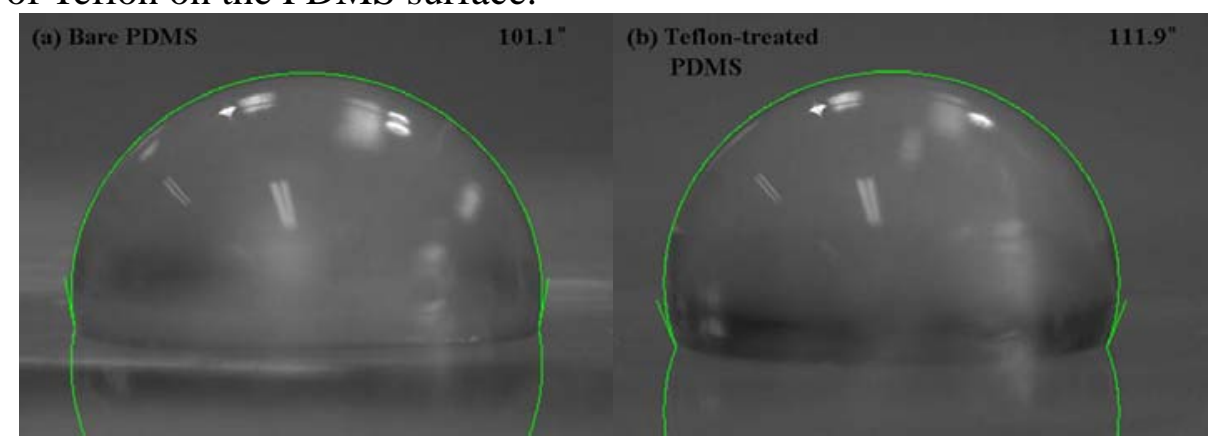

Fig. 2 Contact angle measurements. Water Contact angles measured on the surfaces of a bare PDMS, and $\mathbf{b}$ Teflon-treated PDMS.

In additional, XPS analysis was conducted using XPS spectra (PHI 5600, Physical Electronics Corp.) to analyze the surface elemental composition. Figure 3a and b shows the XPS analysis results of bare PDMS and Teflon-treated PDMS, respectively. The overall element peaks detected on the surfaces as well as corresponding atomic concentrations were presented. It can be found that after surface treatment using Teflon, the element $\mathrm{F}$ appears with a relatively high concentration. It is known that PDMS is a polymer mainly composed of $\mathrm{C}, \mathrm{Si}, \mathrm{H}$, and $\mathrm{O}$, and only Teflon contains $\mathrm{F}$, thus we could conclude that there could be a residual Teflon layer remaining on the surface of PDMS. 

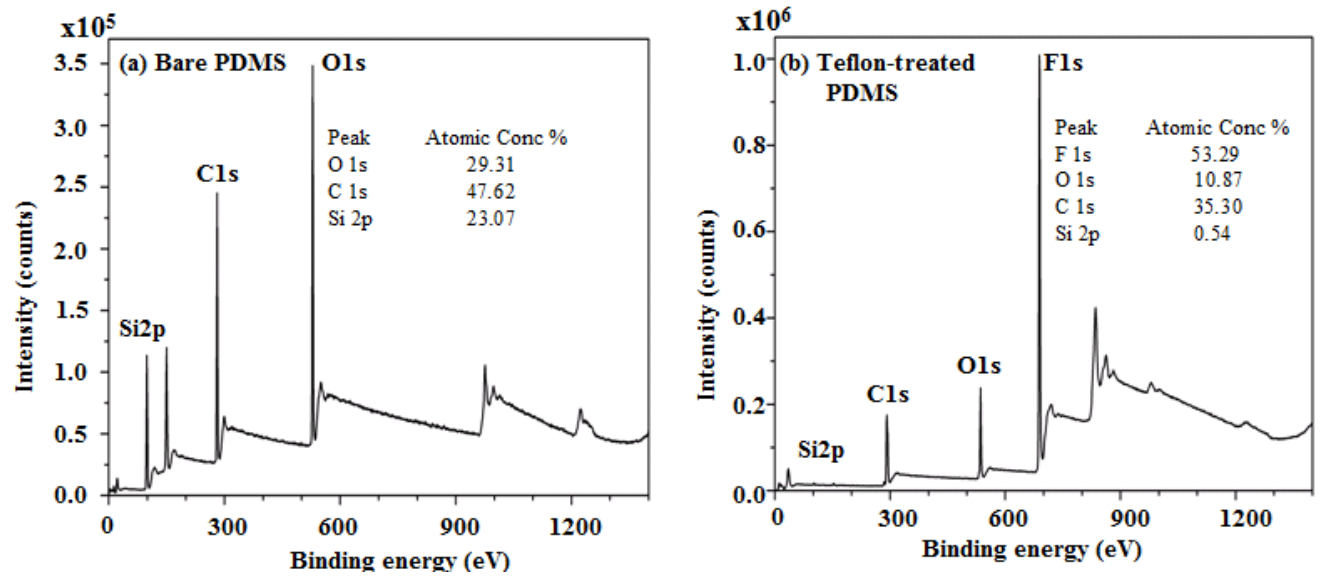

Fig. 3 XPS analysis results of a bare PDMS, and $\mathbf{b}$ Teflon-treated PDMS.

\section{Controlling the thickness of the transferred PDMS layer}

After the transferring PDMS layer was surface-treated with Teflon, PDMS prepolymer was spun on its surface using a spin coater. By varying the spin speed, transferred PDMS layers of different thickness can be achieved. Since the transferred PDMS layer is laser-patterned and serves as a working part of the microfluidic chip, its thickness will affects the performance of the device. Various spin speed as well as corresponding thickness of cured transferred PDMS layers were tested. Figure 4 shows the effect of spin speed on the thickness of the transferred layer. As can be seen, by increasing the spin speed from 500 to 5,000 rpm, the thickness of the transferred PDMS layer decreased from about $220 \mu \mathrm{m}$ to around $20 \mu \mathrm{m}$. This feature enables the fabrication of complicated microfluidic devices having multiple layers of various depths.

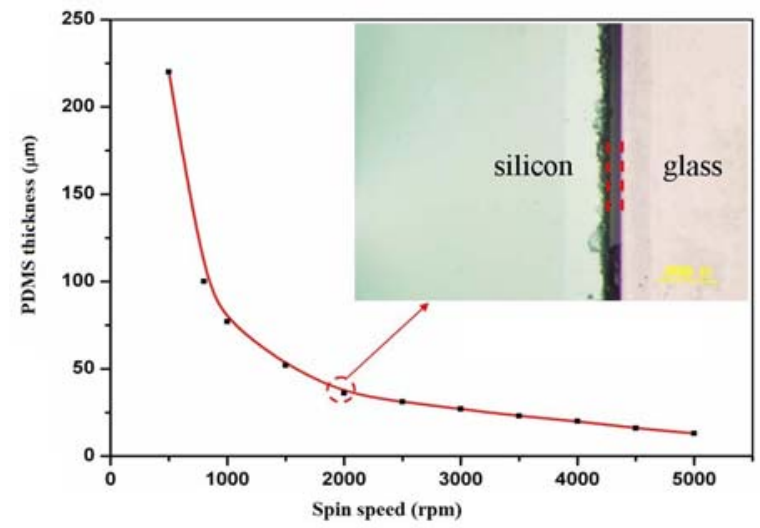

Fig. 4 Picture showing the effect of spin speed on the thickness of transferred PDMS layer. PDMS thickness versus spin speed with the spin time fixed at 30s, the inset picture is a microscopic photo showing a $36 \mu \mathrm{m}$-thick PDMS layer prepared by spinning at 2,000rpm.

\section{Optimization of laser parameters}

There are three main parameters controlling the settings of the laser-cutting machine: laser power, scanning speed and pulses per inch (PPI), which work together to determine the depth and smoothness of cut patterns. Different combinations of these three parameters can result in different spatial printing resolutions. The relation between the laser parameters and cutting profile of 
PDMS pattern was studied by Liu and Gong (2009). These three parameters of pulsed $\mathrm{CO}_{2}$ laser were optimized for cutting PDMS layers of different thickness, and required patterns with ideal profile were formed if they were set to a suitable level. In order to optimize parameters of laser cutting, various tests with different settings of parameters were conducted. Four cutting profiles of lines on a thin PDMS film with a thickness of $36 \mu \mathrm{m}$ were shown in Fig. 5. It is found that the fourth setting is more suitable, by which a line with smoother edge and fewer dust residue was obtained. Although the resolution of $\mathrm{CO}_{2}$ laser cutting is not as high as that of photolithograph using expensive and hard chromium masks, it is comparable to prototyping processes using printed transparent masks and adequate for the fabrication of microfluidic devices in many applications (Snakenborg et al. 2004). In addition, compared with conventional soft lithography process required to construct patterns on PDMS, the laser cutting method is simple, fast and low cost, which show great advantages in microfluidic chips fabrication.

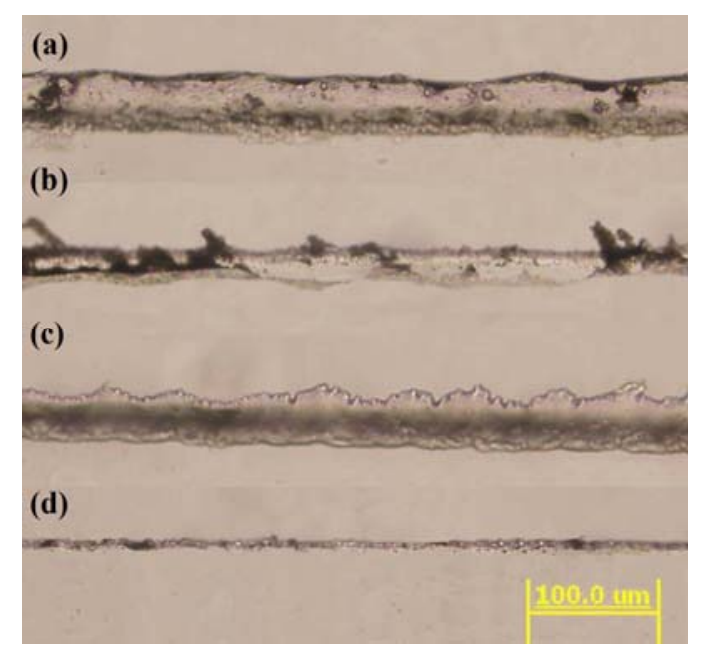

Fig. 5 Microscopic pictures showing the influence of laser parameters on cutting profiles. Lines were cut on a $36 \mu \mathrm{m}$-thick PDMS film by setting laser power, scanning speed and PPI at a $0.25 \mathrm{~W}$, $2.25 \mathrm{~mm} / \mathrm{s}, 1,000 ; \mathbf{b} 0.50 \mathrm{~W}, 0.75 \mathrm{~mm} / \mathrm{s}, 1,000$; $0.25 \mathrm{~W}, 0.75 \mathrm{~mm} / \mathrm{s}, 500$; and d $0.25 \mathrm{~W}, 0.75 \mathrm{~mm} / \mathrm{s}$, 1,000 .

\section{Bonding examination and testing}

The flexibility and compatibility of PDMS enables the creation of a very conform contact to substrates, as silicon, glass or another piece of PDMS, even those having non-uniform topographies. After treatment with oxygen plasma, Si-O-Si bonds are created and irreversible assembly is obtained. In order to study the bonding of the transferred PDMS layer and substrates surface-treated by oxygen plasma, two different material substrates (glass and silicon) were bonded together with a thin patterned layer of PDMS using our developed method. The bonded microstructures were examined under a scanning electron microscope (SEM, JSM-6300). The cross section of the sample was sputter-coated with gold prior to the SEM imaging. Figure 6a and $\mathrm{b}$ shows the bonding of glass-PDMS-glass and glass-PDMS-silicon, respectively. The thickness of the PDMS layer is $36 \mu \mathrm{m}$ and the width of the channel contained in the PDMS layer is $800 \mu \mathrm{m}$. It can be found that the patterned thin PDMS layer can be well bonded to glass and silicon using above-mentioned fabrication process. 


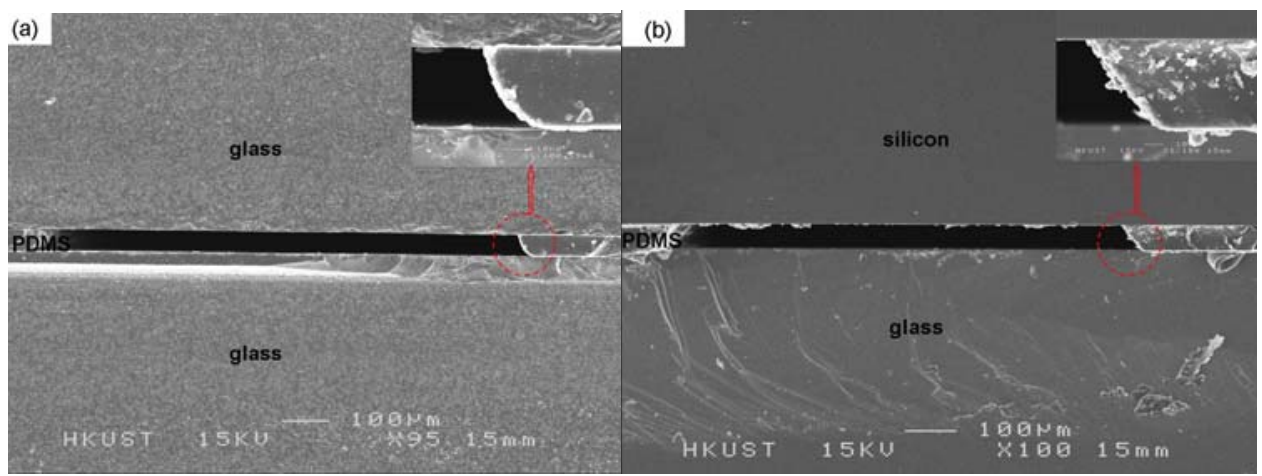

Fig. 6 SEM images of bonding a glass-PDMS-glass, b silicon-PDMS-glass.

The bonding strength was examined by a simple leakage testing, which was performed by introducing an ink solution at various flow rates. A PMDS layer containing a microchannel was sandwiched between two substrates (both glass and silicon substrates have been tested). Two holes serving as fluid inlet and outlet were drilled in the top substrate. Fittings and Teflon tubings (Tygon Tubing) were connected to the tested devices using a UV curable adhesive (NEA 121, Norland Products, NJ, USA). Red ink was employed for visualization. A syringe pump (kdScientific, 781200) was used to introduce ink solution to channel with five selected rates of $0.001,0.01,0.1,1$ and $10 \mathrm{ml} / \mathrm{h}$. For any case of flow rate, no leakage was observed in our experiment.

In addition, the pressure that the chip can sustain without failure by rupture or delamination of intermediate PDMS layer and top or bottom substrate is one important indicator of robustness of the fabrication process and chip operation. The bonding strength testing was conducted using a similar method reported by Eddings et al. (2008). A digital pressure sensor 'Druck DPI 104' (GE Druck, Leicester, UK) was employed to monitor the applied pressure. A hole with a diameter of $1 \mathrm{~mm}$ was drilled through the top substrate for connection to a gas tank which acted as the pressure source. The value of applied pressure increased until the sample is broken, while the value of the pressure breaking structures was recorded. It is found that both glass-PDMS-glass and glass-PDMS-silicon chips remained as one integral unit when the pressure was increased up to $200 \mathrm{kPa}$, which are close to the results of the oxygen-plasma bonding of PDMS represented in that previous reference. This has demonstrated that the developed bonding method is adequate for microfluidic applications.

\section{An integrated electronic-microfluidic device}

Using our developed fabrication method, a microfluidic chip with electrodes on the top and bottom surfaces was fabricated and its function for microparticle manipulation was demonstrated. The theory of this device relies on dielectrophoresis (DEP), which is the resultant movement of particles in a nonuniform electric field due to the polarization effect, and can be compatible with microfluidic systems for wide applications (Kang and Li 2009; Kang et al. 2008; Khoshmanesh et al. 2010; Pethig 2010). Accompanied by the advance in DEP analysis approach (Song and Bennett 2010; Ai et al. 2010; Chen et al. 2005), DEP has draw special attraction of researchers. One method for generating required spatial gradient of electric field is applying alternative current (AC) voltages via electrodes incorporated on chips. The reported fabrication process of AC DEP 
devices comprising three-dimensional (3D) microelectrode configurations generally involves: manufacturing microfluidic channel structure of SU-8 photoresist and application of a UV curable adhesive (Dürr et al. 2003; Cheng et al. 2007) or an epoxy-based adhesive for bonding (Chen et al. 2006), which required standard photolithograph technique to pattern microfluidic channels and exact control of the thickness of adhesive to avoid contamination of the channel. Such fabrication process is relatively complicated, expensive, and timeconsuming.

As an alternative, a laser-patterned PDMS layer containing a microfluidic channel served as an adhesive layer, and was sandwiched between two glass substrates with sputter-coated electrodes after oxygen-plasma treatment. The fabrication process is illustrated in Fig. 7. Patterned HPR 507 was generated on both the top and bottom substrates using the standard photolithograph technique. The electrode layer of $10 \mathrm{~nm} \mathrm{Ti} / 100 \mathrm{~nm} \mathrm{Pt}$ was then deposited and patterned with the lift-off process (see Fig. 8). Due to the flexibility of PDMS, electrodes with a thickness at a nanometer level can be patterned on the substrate without affecting the bonding efficiency. Two holes with the same diameter of $1 \mathrm{~mm}$ were drilled in the top substrate. By setting laser power, scanning speed and PPI at $0.25 \mathrm{~W}, 0.75 \mathrm{~mm} / \mathrm{s}$ and 1,000, respectively, an excellent channel configuration with $36 \mu \mathrm{m}$ depth and 800 $\mu \mathrm{m}$ width was obtained. The transferred PDMS layer containing a channel for fluid confinement was bonded with the electrode-coated substrates after oxygenplasma treatment. Figure 9 shows the completed microfluidic microsystem comprising 3D electrodes fabricated using the above process.

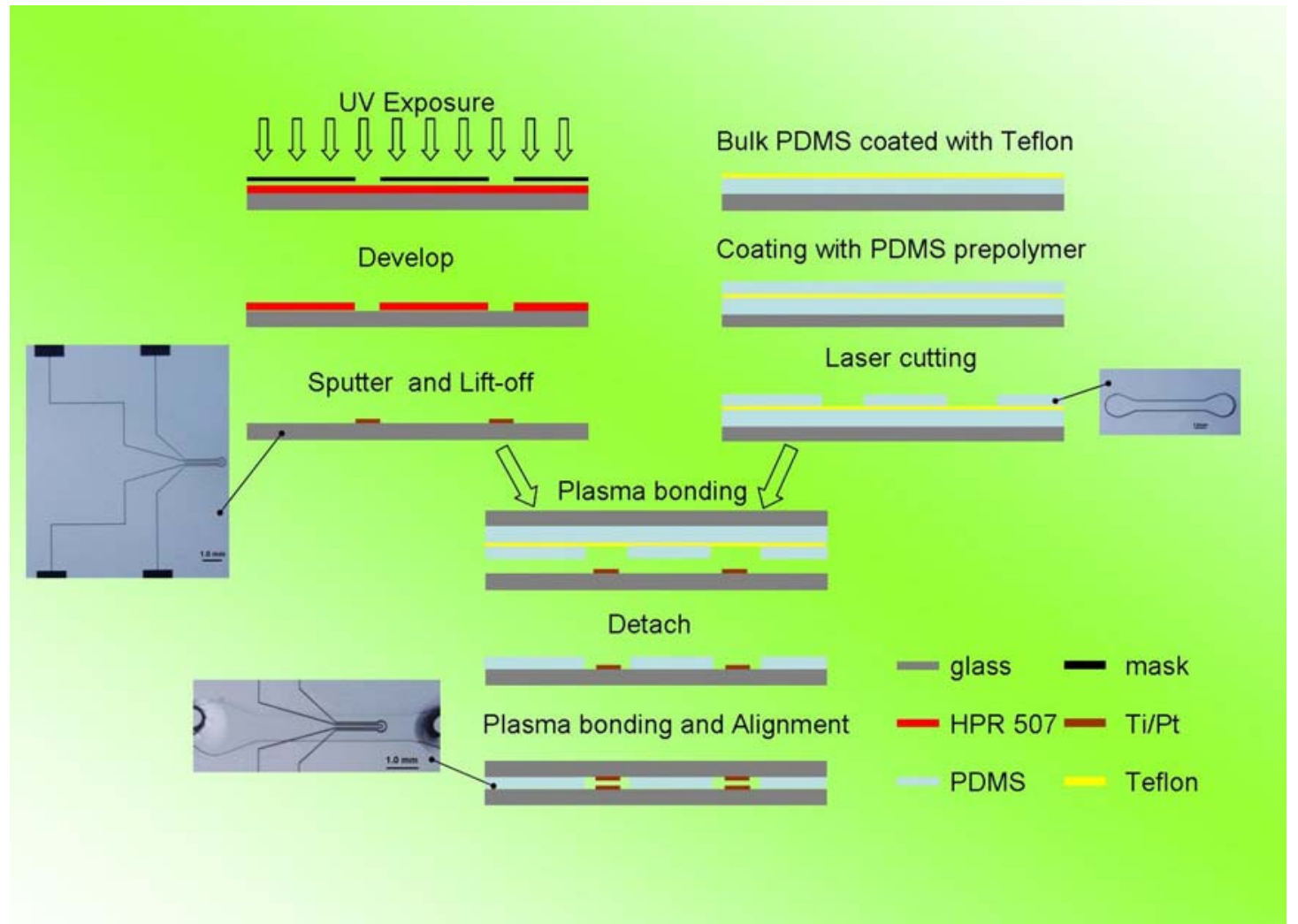

Fig. 7 Schematic view of the fabrication process flow for an integrated electronic-microfluidic device. Insets show top view of sputter-coated electrodes, laser-cut microchannel and final fabricated chip (diagram not to scale) 


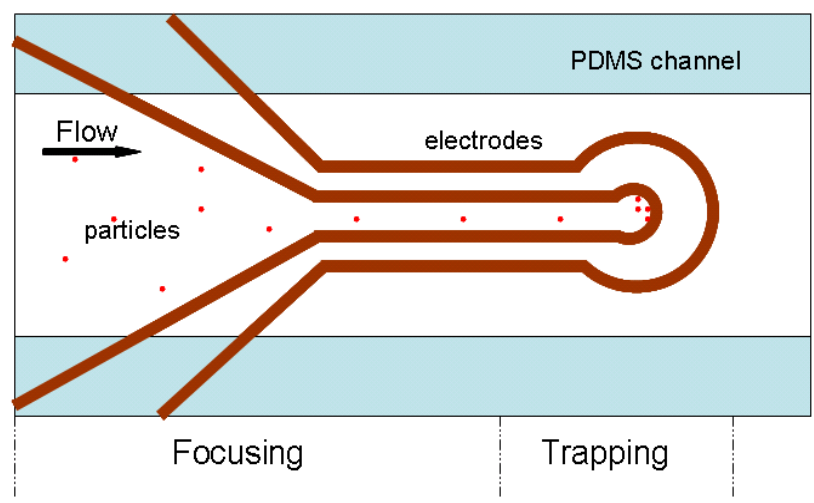

Fig. 8 Diagram of the electrodes sputter-coated on the glass substrate, which consisted of two units: focusing and trapping (wiring connections to external power supply are not shown)

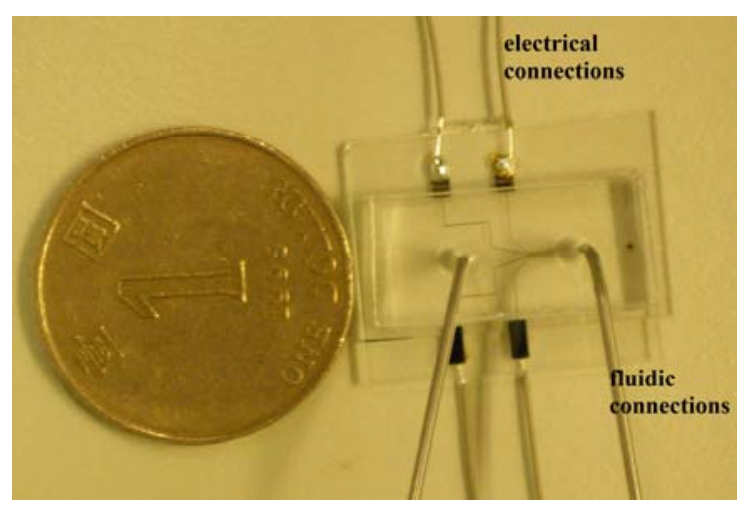

Fig. 9 Photograph of microfabricated microchannel structure with integrated 3D electrodes. Two glass substrates containing electrodes were bonded together by a laser-patterned PDMS layer. Wires and tubings were joined for electrical and fluidic connections, respectively.

The performance of the fabricated chip was evaluated by dielectrophoretically focusing and trapping red fluorescent polystyrene spheres with a diameter of 10 $\mu \mathrm{m}$ (Invtrogen, CA, USA). Before use, the particles were re-suspended in DI water at a final concentration of $10^{5}$ beads $/ \mathrm{ml}$. The sample was injected continuously into the microchannel by a syringe pump (kdScientific, 781200). A function generator (HP 33120A) was used for voltage supply. The traveling of the microparticles was monitored under a microscope (Olympus 1x71, Tokyo, Japan), and recorded by a CCD camera (Olympus DP70). When an electric field was applied, the motion of a particle in the flow is determined by the combined effect of two forces: DEP force and hydrodynamic force. The theory of this experiment was similar to that described by Chen et al. (2006). In our test, particles were first focused by the funnel structure, after which they could move in a narrow stream in the middle of the channel, and finally they were trapped by aggregating at the edge of the arc electrodes. Figures 10 and 11 show the movement of particles at the flow rate of $0.03 \mathrm{ml} / \mathrm{h}$ from left to right with a $10 \mathrm{KHz}, 10 \mathrm{~V}_{\mathrm{p}-\mathrm{p}}$ sinusoidal voltage applied, at which particles experienced negative DEP. It can be found that, particles were uniformly distributed in the microchannel when no electric field was applied (Fig. 10a); in contrast, particles were focused to move in a narrow stream in the middle of channel with the applied AC electric field (Fig. 10b). Figure 11a-d shows that particles were successfully trapped by the arc electrodes. The number of aggregated particles increased along with time variation. In our experiment, no leakage was observed when the fluid flow rate was increased from 0.001 to $10 \mathrm{ml} / \mathrm{h}$ and the maximum pressure in the channel can reach as high as 
$200 \mathrm{kPa}$. Depending on the application of electrodes patterned the top and bottom of microchannel, the fabricated microfluidic structure accomplished dielectrophoretic focusing and trapping of microparticles, which has potential applications in continuous bioparticle manipulation and flow cytometry. It is proved that integrated microfluidic structures manufactured by our developed method have electrical characteristics needed for use in microanalytical systems.
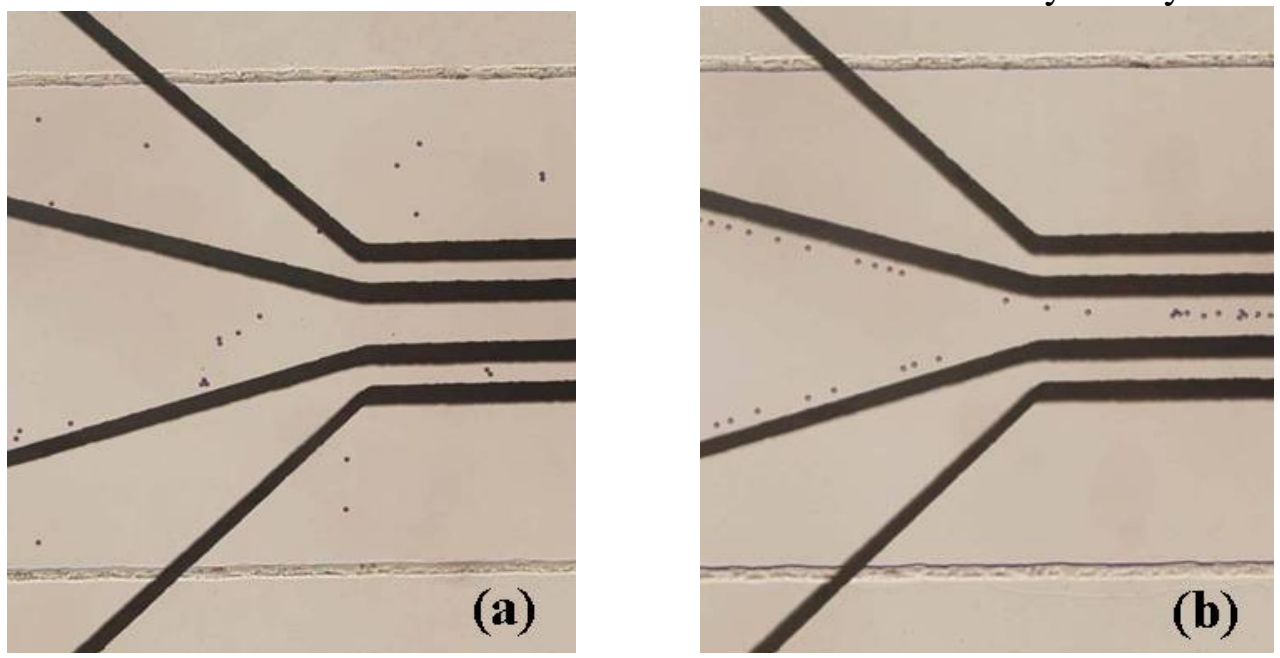

Fig. 10 Pictures showing microparticles $(10 \mu \mathrm{m})$ were focused by slanted electrodes. a Microparticles were distributed uniformly without electric filed. b Microparticles moved in the middle of the channel when applying an AC electric field at $10 \mathrm{kHz}$ and $10 \mathrm{~V}_{\mathrm{p}-\mathrm{p}}$.

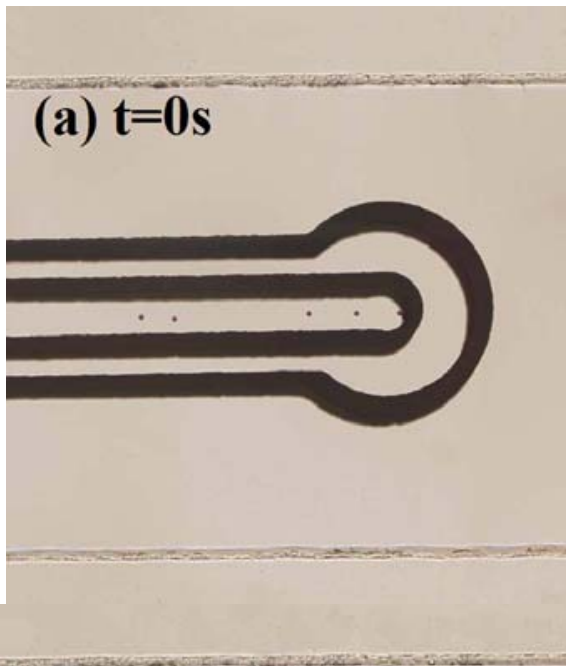

(c) $\mathbf{t}=\mathbf{2 0 s}$

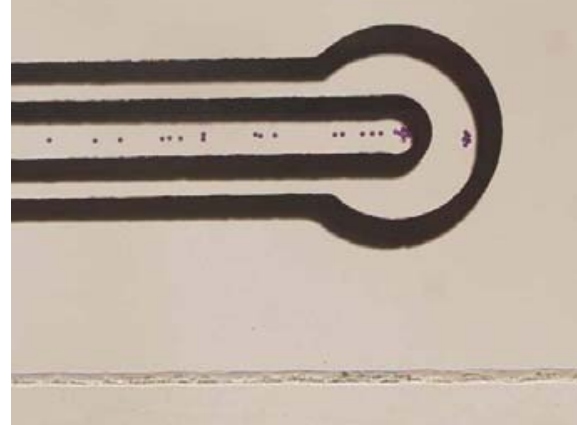

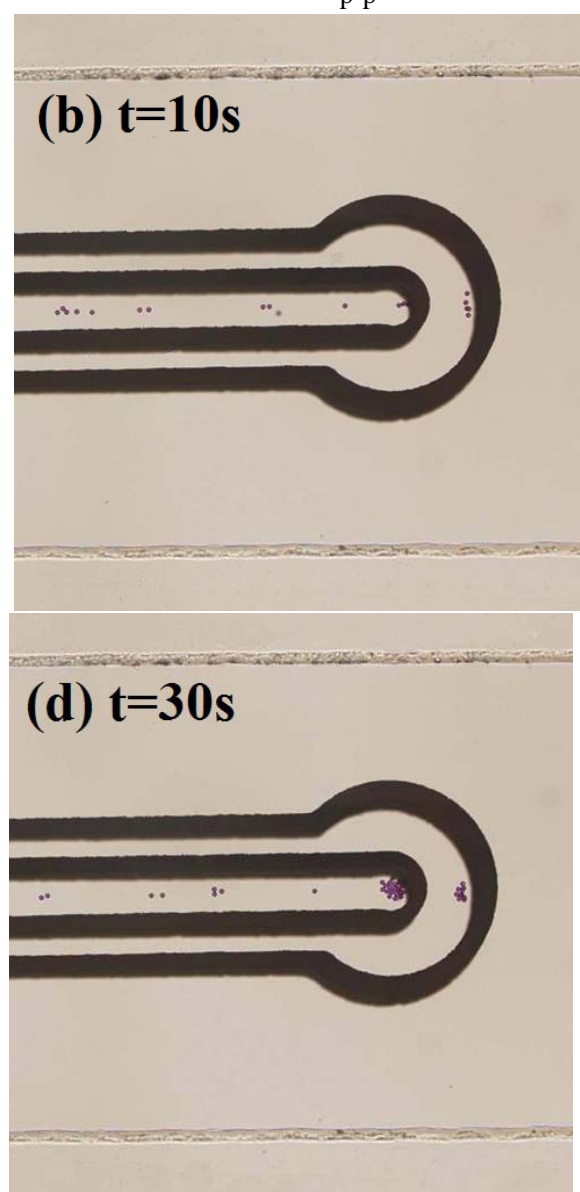

Fig. 11 Pictures showing microparticles $(10 \mu \mathrm{m})$ were trapped by arc electrodes. a $\mathrm{t}=0 \mathrm{~s}, \mathbf{b} \mathrm{t}=10 \mathrm{~s}$, c $\mathrm{t}=20 \mathrm{~s}, \mathbf{d} \mathrm{t}=30 \mathrm{~s}$. 


\section{Conclusions}

In this work, we developed a simple and cost-effective approach for the fabrication of integrated microfluidic devices having multilayer structures. With this method, each distinct layer-transferred PDMS layer, top and bottom substrate was processed individually: laser cutting technique was applied on transferred PDMS layers to construct microchannels, while lithography, sputtering and lift-off techniques were applied on substrates for surface patterning. After oxygen-plasma treatment, distinct layers were bonded together to form an enclosed integrated microfluidic microsystem for various applications. The advantages of the developed fabrication method involve: (1) complicated and laborious soft lithography processes for template fabrication, generally required to be conducted in a high-standard clean room were avoided, which thus can greatly reduce the fabrication time and save the fabrication costs; (2) incorporating micromachined structures with electronics on top and bottom surfaces of microfluidic channels was easily achieved for increased functionality. Therefore, the fabricated microfluidic device can be considered as a complex microsystem including mechanical, electronic, fluid functions, etc.; (3) integrated multilayer microfluidic devices, such as those with multiple channels of different configurations or depths can be obtained by transfer-bonding many laser-patterned PDMS layers together; (4) design and surface modification of microfluidic devices were diversified, because it gets rid of strict fabricating conditions, such as high temperature and voltage, ultra-clean and ultra-flat surface, and specific chemical compositions. The validation of this method was illustrated by fabricating and testing a DEPbased integrated electronic-microfluidic chip for microparticle manipulation. The proposed simple and cost-effective fabrication method is expected to be widely used for manufacturing integrated multilayer microfluidic devices combining electrical and microfluidic functions.

\section{Acknowledgements}

The author (W.H. Li) wishes to thank Australian government to offer an Australian Endeavour Research Fellowship (1994_2011) to enhance research collaboration with Prof. W. Wen. This project is also partially supported by University of Wollongong through a UIC grant.

\section{References}

Ai Y, Park S, Zhu J, Xuan X, Beskok A, Qian S (2010) DC electrokinetic particle transport in an L-shaped microchannel. Langmuir 26(4): 2937-2944

Alexe M, Gösele U (2004) Wafer bonding: applications and technology. Springer, Berlin

Chen DF, Du H, Li WH, Shu C (2005) Numerical modeling of dielectrophoresis using a meshless approach. J Micromech Microeng 15: 1040-1048

Chen DF, Du H, Li WH (2006) A 3D paired microelectrode array for accumulation and separation of microparticles. J Micromech Microeng 16: 1162-1169

Cheng I-F, Chang H-C, Hou D, Chang H-C (2007) An integrated dielectrophoretic chip for continuous bioparticle filtering, focusing, sorting, trapping, and detecting. Biomicrofluidics 1: 021503. doi: 10.1063/1.2723669

Cheng Y, Sugioka K, Midorikawa K (2004) Microfluidic laser embedded in glass by threedimensional femtosecond laser microprocessing. Opt Lett 29(17): 2007-2009

Cheng Y, Xu J, Xu Z, Sugioka K, Midorikawa K (2008) Femtosecond laser integration for biophotonic applications-A "magic brush" in the micro/nano-world. In: Optical fiber communication and optoelectronic exposition and conference 2008. AOE 2008, Asia

Dürr A, Kentsch J, Muller T, Schnelle T, Stelzle M (2003) Microdevices for manipulation and 
accumulation of micro- and nanoparticles by dielectrophoresis. Electrophoresis 24: 722-731

Eddings MA, Johnson MA, Gale BK (2008) Determining the optimal PDMS-PDMS bonding technique for microfluidic devices. J Micromech Microeng 18: 067001. doi:10.1088/0960$1317 / 18 / 6 / 067001$

El-Ali J, Sorger PK, Jensen KF (2006) Cells on chips. Nature 442: 403-411

Ford SM, Davies J, Kar B, Qi SD, Mcwhorter S, Soper SA, Malek CK (1999) Micromachining in plastics using X-ray lithography for the fabrication of micro-electrophoresis devices. J Biomech Eng 121(1): 13-21

Harrison DJ, Manz A, Fan Z, Lüdi H, Widmer HM (1992) Capillary electrophoresis and sample injection systems integrated on a planar glass chip. Anal Chem 64(17): 1926-1932

Iyer SS, Auberton-Hervé AJ (2002) Silicon wafer bonding technology for VLSI and MEMS. INSPEC, London

Jacobson SC, Hergenröder R, Koutny LB, Warmack RJ, Ramsey JM (1994) Effects of injection schemes and column geometry on the performance of microchip electrophoresis devices. Anal Chem 66(7): 1107-1113

Kang Y, Li D, Kalams SA, Eid JE (2008) DC-dielectrophoretic separation of biological cells by size. Biomed Microdev 10: 243-249

Kang Y, Li D (2009) Electrokinetic motion of particles and cells in microchannels. Microfluid Nanofluid 6: 431-460

Khoshmanesh K, Nahavandi S, Baratchi S, Mitchell A, Kalantar-zadeh K (2010) Dielectrophoretic platforms for bio-microfluidic systems. Biosens Bioelectron 26(5): 1800-1814

Kovacs GTA (1998) Micromachined transducers sourcebook. McGraw-Hill, New York

Liu H-B, Gong H-Q (2009) Templateless prototyping of polydimethylsiloxane microfluidic structures using a pulsed CO2 laser. J Micromech Microeng 19: 037002. doi:10.1088/09601317/19/3/037002

Maharbiz MM, Cohn MB, Howe RT, Horowitz R, Pisano AP (1999) Batch micropackaging by compression-bonded wafer-wafer transfer. Proceedings of MEMS Orlando, FL, USA, pp 482-489. doi: 10.1109/MEMSYS.1999.746876

McDonald JC, Duffy DC, Anderson JR, Chiu DT, Wu H, Schueller OJA, Whitesides GM (2000)

Fabrication of microfluidic systems in poly(dimethylsiloxane). Electrophoresis 21: 27-40

Pethig R (2010) Review article-dielectrophoresis: status of the theory, technology, and applications. Biomicrofluidics 4: 022811. doi:10.1063/1.3456626

Plecis A, Chen Y (2007) Fabrication of microfluidic devices based on glass-PDMS-glass technology. Microelectron Eng 84: 1265-1269

Quake SR, Scherer A (2000) From micro- to nanofabrication with soft materials. Science 290: 1536-1540

Roberts MA, Rossier JS, Bercier P, Girault H (1997) UV laser machined polymer substrates for the development of microdiagnostic systems. Anal Chem 69(11): 2035-2042

Schmidt H, Ihlemann J, Luther K, Troe J (1999) Modeling of velocity and surface temperature of the moving interface during laser ablation of polyimide and poly (methylmethacrylate). Appl Surf Sci 138-139: 102-106

Schmidt MA (1998) Wafer-to-wafer bonding for microstructure formation. Proc IEEE 86(8): 1575-1585

Snakenborg D, Klank H, Kutter JP (2004) Microstructure fabrication with a $\mathrm{CO}_{2}$ laser system. J Micromech Microeng 14: 182-189

Song H, Bennett DJ (2010) A semi-analytical approach using artificial neural network for dielectrophoresis generated by parallel electrodes. J Electrostat 68: 49-56

Sparks D, Queen G, Weston R, Woodward G, Putty M, Jordan L, Zarabadi S, and Jayakar K (2001) Wafer-to-wafer bonding of nonplanarized MEMS surfaces using solder. J Micromech Microeng 11: 630-634

Tong Q-Y, Gösele U (1999) Semiconductor wafer bonding: science and technology. Wiley, New York

Ueno K, Kitagawa F, Kim H-B, Tokunaga T, Matsuo S, Misawa H, Kitamura N (2000) Fabrication and characteristic responses of integrated microelectrodes in polymer channel chip. Chem Lett 29(8): 858-859

Whitesides GM (2006) The origins and the future of microfluidics. Nature 442: 368-373

Wu H, Huang B, Zare RN (2005) Construction of microfluidic chips using polydimethylsiloxane for adhesive bonding. Lab Chip 5: 1393-1398

Wu H, Odom TW, Chiu DT, Whitesides GM (2003) Fabrication of complex three-dimensional microchannel systems in PDMS. J Am Chem Soc 125(2): 554-559

Xia Y, Whitesides GM (1998) Soft lithography. Annu Rev Mater Sci 28: 153-184

Zhang M, Wu J, Wang L, Xiao K, Wen W (2010) A simple method for fabricating multi-layer 
PDMS structures for 3D microfluidic chips. Lab chip 10: 1199-1203 